\title{
Brain Mechanisms of College Students' Social Adjustment: Evidence from Multimodal Magnetic Resonance Imaging (MRI)
}

\author{
Ying Ge ${ }^{1,2 *}$, Weigang Pan²* Tin Wang ${ }^{1,2}$ \\ ${ }^{1}$ Mental Health Education Research Center, Southwest University, Chongqing, China \\ ${ }^{2}$ Key Laboratory of Emotion and Mental Health in Chongqing, Chongqing University of Arts and Sciences, \\ Chongqing Collaborative Innovation Center for Brain Science, Chongqing, China \\ Email: ^gy8620@163.com, ${ }^{\star} 2998623986 @ q q . c o m$
}

How to cite this paper: Ge, Y., Pan, W.G. and Wang, T. (2018) Brain Mechanisms of College Students' Social Adjustment: Evidence from Multimodal Magnetic Resonance Imaging (MRI). Health, 10, 442-457. https://doi.org/10.4236/health.2018.104036

Received: March 12, 2018

Accepted: April 24, 2018

Published: April 27, 2018

Copyright $\odot 2018$ by authors and Scientific Research Publishing Inc. This work is licensed under the Creative Commons Attribution International License (CC BY 4.0).

http://creativecommons.org/licenses/by/4.0/

(c) (i) Open Access

\begin{abstract}
This study investigated the neural basis of social adjustment using multimodal brain imaging and social-adjustment measurements to analyze functional and structural brain features during social adjustment in college students. The results showed that, regarding brain function, some dimensions of social adjustment were associated with the insula, and some regions of the frontal and occipital lobes. Self-adjustment and satisfaction required activation of the middle frontal gyrus, while career adjustment and academic adjustment required inhibition of the inferior frontal gyrus and lingual gyrus, respectively. Decreased metabolic activity of the lingual gyrus was beneficial for obtaining satisfaction. Regarding brain structure, the total score and some dimensions of social adaptation were associated with the gray matter of portions of the temporal and parietal lobes. The superior temporal gyrus was associated with the total social adjustment and satisfaction score, the middle temporal gyrus with campus-life adjustment and satisfaction, and the post central gyrus and the inferior parietal lobule with emotional adjustment. The changes in the gray matter volume of these brain regions to a certain extent reflected socially adaptive behaviors. The results suggest that social adaptability is associated with various brain regions dispersed among both hemispheres of the brain, and requires synergistic interactions between multiple brain regions and both brain hemispheres.
\end{abstract}

\section{Keywords}

Social Adjustment, Multimodal Neuroimaging, Resting-State Functional Magnetic Resonance Imaging, Structural Magnetic Resonance Imaging 


\section{Introduction}

Adjustment is a biological term used to denote the physical and behavioral changes that can increase the survival opportunity of an organism. In psychology, it is used to represent human reactions to environmental changes, wherein adjustment to the social environment is called "social adjustment". Social adjustment is a core component of mental health: It is a positive and active reaction the subject makes through his or her self-regulation system, which allows the continuous formation of new equilibriums between the subject and the social environment [1]. Therefore, adjustment, and particularly social adjustment, is an important feature of mental health, and a life issue that an individual must face and resolve. College students are in late puberty, which is an important period in an individual's development. During this stage, physical and mental activities undergo significant changes, and the abilities of self-regulation and self-control are not strong. Thus, in the current complex transitional period of Chinese society, when college students face various pressures, they often experience psychological disorders and maladjustments. Regarding social adjustment, studies outside of Chinese society have focused on cultural adaptation to the broader social environment and background, as well as stress adaptation in emergencies, but studies of the specific social adjustment of college students are few in number [2] [3], and only a small number of studies have addressed adolescent adaptation [4] [5]. Studies of the social adjustment of undergraduate students in China have primarily focused on the adjustment of freshmen after entering college and the social adjustment of new graduates. Research topics have included the concept, structure, and evaluation tools pertaining to college students' social adaptability, and relevant influencing factors [6]-[11]. Exploration of the brain mechanisms underlying social adjustment needs consideration.

In recent years, the development of multimodal brain imaging techniques has provided the technical tools and research basis for exploring functional and structural brain features. As a relatively stable psychological property, the neural mechanisms of social adaptation may be associated with resting-state brain activity and brain structures. Resting-state functional magnetic resonance imaging (rs-fMRI) primarily detects spontaneous brain activity of individuals in the absence of tasks, while regional homogeneity (ReHo) is an imaging parameter based on rs-fMRI that investigates the consistency of spontaneous neural activity and function in brain regions [12]. It is also possible to analyze brain tissue volume and density using structural magnetic resonance imaging (sMRI). Voxel-based morphometry (VBM) analyzes brain structure images at the voxel level. It can facilitate quantitative comparisons of differences in density and volume of gray matter and white matter, thereby accurately showing changes in brain morphology [13] [14]. Correlation analysis of individual behaviors with imaging-derived functional and structural data is a new approach to studying the brain mechanisms underlying individual behaviors. Currently, many researchers 
use resting-state functional and structural MRI technology to study how psychological properties, such as personality of normal individuals, are reflected in the brain function [15] [16] [17], and the relationship between these properties and brain structure [18] [19] [20].

Here, we used multimodal brain imaging techniques and social adaptation measurements to explore the relationship between college students' social adjustment and brain function and structure, and provided an initial insight into the neural mechanisms of social adjustment.

\section{Method}

\subsection{Participants}

The inclusion criteria for participants in this study include the following: 1) All participants are college students; 2) They all report no history of physical or mental illness; 3) Their vision or corrected vision is normal. In terms of the criteria, fifty college students were initially recruited for this study. After excluding unqualified data, 41 individuals finally participated (average age: 20.01 years; 14 males and 27 females). Before the experiment, the participants were informed about the research processes and details, and after receiving this information, each participant voluntarily signed the informed consent form. After completion of the study, each participant received compensation (50 RMB). All experimental procedures and processes were reviewed by the academic ethics committee.

\subsection{Research Design}

The Chinese College Student Adjustment Scale (CCSAS) [21] was used to measure the social adaptability of individuals, ReHo analysis (regional homogeneity) was used to assess the individual's functional brain characteristics, and brain tissue volume analysis obtained by VBM (voxel-based morphometry) reflected the structural properties of the individual brain.

The CCSAS consists of 60 items, which can be scored positively or negatively, encompassing seven dimensions, namely: interpersonal adjustment, academic adjustment, campus life adjustment, career adjustment, emotional adjustment, self-adjustment, and satisfaction. The coefficient of internal homogeneity of the scale $(\alpha)$ is 0.93 , and the test-retest reliability coefficient is 0.99 . The score of the scale ranges from 1 to 5 , whereby higher scores denote better adaptability.

ReHo values measure the similarity of the time series of a given voxel to its neighboring voxels, which reflect, from the perspective of regional homogeneity, synergy among blood oxygen level-dependent signals in local brain regions during spontaneous neural activity. VBM analyzes high-resolution TI images, and quantitatively calculates the signal strength within local gray and white matter voxels. The multiple regression method provided by SPM8 was used to model the relationship between individual behavior and brain functional and structural differences, thus exploring the functional and structural cortical basis of social adjustment. 


\subsection{Data Collection}

A 3.0T MRI system (MAGNETOM Trio Tim, Siemens, Shanghai, China) was used to collect data. During the resting-state scan, the head of the tested subject was stabilized using foam pads, and earplugs were used to reduce interference due to scanner noise. The subject lay flat on his or her back with eyes closed and with no particular mental activity specified [22].

Functional imaging data were obtained by whole-brain gradient-echo echo-planar imaging (EPI). The parameters were as follows: total scanned layers = 32 , repetition time $(\mathrm{TR})=2000 \mathrm{~ms}$, echo time $(\mathrm{TE})=30 \mathrm{~ms}$, flip angle $(\mathrm{FA})=$ $90^{\circ}$, field of view $(\mathrm{FOV})=240 \mathrm{~mm}$ ) of $\mathrm{mm}$, scanning matrix $=64 \mathrm{sca} \mathrm{mm}$, slice thickness $=3 \mathrm{~mm}$, slice gap $=1 \mathrm{~mm}$, voxel size $=3.4 \times 3.4 \times 4 \mathrm{~mm}^{3}$. The total scanning time was 8 minutes, and consecutive images from 240 time points were obtained. High-resolution T1-weighted structural imaging data were obtained using a magnetization-prepared rapid gradient-echo sequence (MPRAGE), with the following parameters: $\mathrm{TR}=1900 \mathrm{~ms}, \mathrm{TE}=2.52 \mathrm{~ms}, \mathrm{FA}=9^{\circ}$, scan matrix $=$ $256 \times 256 \mathrm{~mm}^{2}$, number of layers $=176$, voxel thickness $=1.0 \mathrm{~mm}$, and voxel size $=1$ xel siz $\mathrm{mm}^{3}$.

\subsection{Data Processing}

\subsubsection{Data Preprocessing}

Functional imaging data were preprocessed with the Matlab-based DPABI software V2.3 [23]. The original DICOM data were changed into NIFTI data format. To minimize the impact of uneven magnetic field and initial adaptation of the participant to the scanner environment, the data of the first 10 time points were removed. For the remaining 230 time points, time layer correction and head movement correction were performed (all data with greater than $3 \mathrm{~mm}$ or $3 \mathrm{de}-$ grees of head movement were removed). The data were also normalized to MNI standard space; the normalized voxel size was $3 \times 3 \times 3 \mathrm{~mm}^{3}$. The linear drift of the data was removed to correct for physiological baseline noise. The data were also processed by Gaussian smoothing $(0.01-0.08 \mathrm{~Hz})$. Finally, regional homogeneity (ReHo) was calculated. Kendall's coefficient of concordance (KCC) was used as the ReHo value of each voxel. KCC is a standardized value, which is obtained by dividing individual voxel values by the average whole-brain ReHo value [12].

Preprocessing of structural imaging data used Matlab 7.8-based (R2009a; Math Works Inc., Natick, MA, USA) Statistical Parametric Mapping software (SPM8; http://www.filjon.ucl.ac.uk/spm) and the Diffeomorphic Anatomical Registration through Exponentiated Lie (DARTEL) algebra toolbox for voxel-based morphometry (VBM) analysis [24] [25]. The specific procedures consisted of deletion of large artifacts or abnormal morphological data of subjects; transformation of the original DICOM data into NIFTI data, incorporating the img and hdr file formats required by SPM; AC-PC (anterior-posterior commissure plan) position correction for individual structural images with larger devia- 
tion of head position [26]; dividing the structural imaging data into gray matter (GM), white matter (WM), and cerebrospinal fluid components using the "new segment" function of SPM8 [27] [28]; obtaining an affine transformation matrix between each image and the standard template, and normalizing images into an identical space via this matrix; normalizing each subject's separate gray matter and white matter data to the MNI template, constructing $1.5 \times 1.5 \times 1.5 \mathrm{~mm}$ voxel samples from the structural images of each subject, and obtaining images with modulated gray matter volume; finally, smoothing images using a Gaussian kernel of FWHM $8 \mathrm{~mm}$ to reduce spatial noise and improve the signal-to-noise ratio.

\subsubsection{Statistical Analysis}

SPSS 18.0 was used to process and analyze the behavioral data of participants.

The relationships between the individual social adjustment score and the ReHo and volume parameters of the structural data were analyzed using multiple regressions in SPM8. In the analysis of brain functional imaging data, individual CCSAS scores were the variable of interest, gender, and age were used as covariates and the analysis was restricted via a gray-matter mask. In the analysis of brain structural imaging data, gender, age and whole-brain volume (the sum of the gray-matter volume, white-matter volume and cerebrospinal fluid) were used as covariates, individual CCSAS scores were the dependent variable, and the analysis was restricted to a gray-matter mask generated from the data of all participants.

In the multiple regression analysis, correction for multiple comparisons was implemented by Monte Carlo simulation with DPABI V2.3. In the analysis of brain functional and structural data, the smoothing kernel was set to $8 \mathrm{~mm}$. Resting-state correction for multiple comparisons was set at the threshold of a voxel-wise $p<0.001$ and a mass $p<0.05$ that belonged to a cluster with an extent of at least 56 voxels. The threshold of VBM correction for multiple comparisons included that $p<0.001$ was set as a single voxel and $p<0.05$ as a mass, the minimum mass of the structural data was greater than 493 voxels.

\section{Results}

\subsection{Behavioral Data}

Table 1 shows that there were no significant differences between males and females $(p>0.05)$ with respect to the dimensions of the social adjustment scale.

\subsection{Functional Imaging Data}

Multiple regression analysis was conducted on ReHo values, CCSAS total scores, and seven relevant dimensions (interpersonal adjustment, academic adjustment, campus life adjustment, career adjustment, emotional adjustment, self-adjustment, and satisfaction). Gender and age were set as covariates. ReHo values were positively correlated with CCSAS dimension scores in the following brain regions: 
Table 1. Gender difference of the social adaptation scale.

\begin{tabular}{cccc}
\hline Dimension & Male (17) & Female (23) & Tvalue \\
\hline Interpersonal adjustment & $32.54 \pm 3.10$ & $30.78 \pm 4.19$ & 1.49 \\
Academic adjustment & $36.08 \pm 3.45$ & $33.74 \pm 4.12$ & 1.88 \\
Campus life adjustment & $26.31 \pm 2.66$ & $26.59 \pm 3.04$ & -0.30 \\
Career adjustment & $29.46 \pm 2.47$ & $29.22 \pm 2.89$ & 0.27 \\
Emotional adjustment & $26.38 \pm 2.87$ & $27.11 \pm 2.94$ & -0.74 \\
Self-adjustment & $28.69 \pm 1.44$ & $26.37 \pm 2.92$ & 3.37 \\
Satisfaction & $17.77 \pm 3.54$ & $16.41 \pm 3.28$ & 1.17 \\
Total score & $21.00 \pm 13.78$ & $20.26 \pm 10.82$ & 0.17 \\
\hline
\end{tabular}

Note: ${ }^{*} p<0.05,{ }^{* *} p<0.01,{ }^{* * *} p<0.001$.

self-adaptation in the left middle frontal gyrus, and satisfaction in the right middle frontal gyrus. In contrast, ReHo values were negatively correlated with CCSAS dimension scores in the following brain regions: academic adaptation in the left lingual gyrus, career adaptation in the left inferior frontal gyrus, and satisfaction in the left insula (Table 2 and Figure 1).

\subsection{Structural Imaging Data}

Multiple regression analysis was conducted to assess the relationships between gray matter volume and the CCSAS total score and scores of the individual CCSAS dimensions. Gender, age and the whole brain volume (sum of the gray matter volume, white matter volume and cerebrospinal fluid) were used as covariates. Gray-matter volume was positively correlated with CCSAS scores in the following brain areas: the total score and the right superior temporal gyrus, campus life adaptation and the right middle temporal gyrus, emotional adaptation and the left posterior central gyrus and the left inferior parietal lobe, and satisfaction and the right superior temporal gyrus and the middle temporal gyrus (Table 3 and Figure 2).

\section{Discussion}

In this study, we used the CCSAS scale to measure various aspects of the social adjustment of individuals, and resting-state functional and structural brain imaging data to measure the brain features of individuals. We then established links between individual social adjustment behavioral measurements and characteristics of the human brain. The results showed that the individual's social adjustment is associated with various brain regions dispersed among both hemispheres, and requires synergistic interactions between these regions and hemispheres.

\subsection{Brain Functional Characteristics of Social Adjustment}

In the prefrontal lobe, self-adjustment and the left middle frontal gyrus, and satisfaction and the right middle frontal gyrus were positively correlated, while 
Table 2. Brain regions that show significant correlation between ReHo value and CCSAS dimensions.

\begin{tabular}{|c|c|c|c|c|c|c|}
\hline \multirow{2}{*}{ Dimensions and brain regions } & \multirow{2}{*}{ Hemisphere } & \multicolumn{3}{|c|}{ MNI axis } & \multirow{2}{*}{$\begin{array}{c}\begin{array}{c}\text { Number of } \\
\text { voxels }\end{array} \\
\text { (voxels) }\end{array}$} & \multirow{2}{*}{$\begin{array}{c}\mathrm{t} \text { value } \\
\text { (maximum } \\
\text { point) }\end{array}$} \\
\hline & & $\mathrm{x}$ & $\mathrm{y}$ & $\mathrm{z}$ & & \\
\hline \multicolumn{7}{|l|}{ Positive correlation } \\
\hline $\begin{array}{l}\text { Self-adjustment and middle } \\
\text { frontal gyrus }\end{array}$ & Left & -21 & 45 & 24 & 52 & 0.60 \\
\hline $\begin{array}{l}\text { Satisfaction and middle } \\
\text { frontal gyrus }\end{array}$ & Right & 3 & 60 & -3 & 60 & 0.61 \\
\hline \multicolumn{7}{|l|}{ Negative correlation } \\
\hline $\begin{array}{c}\text { Academic adjustment and } \\
\text { lingual gyrus }\end{array}$ & Left & -18 & -66 & -6 & 61 & -0.56 \\
\hline $\begin{array}{l}\text { Career adjustment and inferior } \\
\text { frontal gyrus }\end{array}$ & Left & -27 & 24 & -6 & 69 & -0.53 \\
\hline Satisfaction and insula & Left & -42 & -3 & -6 & 65 & -0.53 \\
\hline
\end{tabular}

Table 3. Brain regions that show significant correlations between the grey matter density and CCSAS dimensions.

\begin{tabular}{|c|c|c|c|c|c|c|}
\hline \multirow{2}{*}{ Dimension and brain region } & \multirow{2}{*}{ Hemisphere- } & \multicolumn{3}{|c|}{ MNI axis } & \multirow{2}{*}{$\begin{array}{c}\text { Number of } \\
\text { voxels }\end{array}$} & \multirow{2}{*}{$\frac{\mathrm{R} \text { value }}{\text { (maximum) }}$} \\
\hline & & $\mathrm{x}$ & $y$ & $\mathrm{z}$ & & \\
\hline \multicolumn{7}{|l|}{ Positive correlation } \\
\hline Total score and superior temporal gyrus & Right & 55.5 & -4.5 & -6.0 & 623 & 3.98 \\
\hline $\begin{array}{l}\text { Campus life adjustment and the } \\
\text { middle temporal gyrus }\end{array}$ & Right & 55.5 & -13.5 & -13.5 & 1326 & 4.68 \\
\hline $\begin{array}{l}\text { Emotional adjustment and the } \\
\text { posterior central gyrus }\end{array}$ & Left & -66.0 & -21.0 & 15.0 & 656 & 4.61 \\
\hline $\begin{array}{l}\text { Emotional adjustment and } \\
\text { inferior parietal lobe }\end{array}$ & Left & -42.0 & -27.0 & 45.0 & 1049 & 5.21 \\
\hline $\begin{array}{l}\text { Satisfaction and the superior } \\
\text { temporal gyrus }\end{array}$ & Right & 58.5 & 12.0 & -1.50 & 1439 & 5.59 \\
\hline Satisfaction and middle temporal gyrus & Right & 64.5 & -30.0 & -27.0 & 703 & 4.52 \\
\hline
\end{tabular}

career adjustment and the left inferior frontal gyrus were negatively correlated. The frontal lobe is responsible for thinking and planning, and is associated with the individual's needs and feelings. The prefrontal cortex is a primary center of emotional regulation, in which the middle frontal gyrus has a top-down, regulatory function, and is associated with memory consolidation and vigilance [29]. Self-adjustment presents as keen self-awareness, i.e., appropriate self-evaluation and positive emotional experiences, while satisfaction is the degree of pleasure felt after an individual's needs are met. Previous resting-state brain functional imaging studies have shown that, compared with controls, individuals with adaptation disorders exhibit abnormal synchronization of spontaneous neuronal 

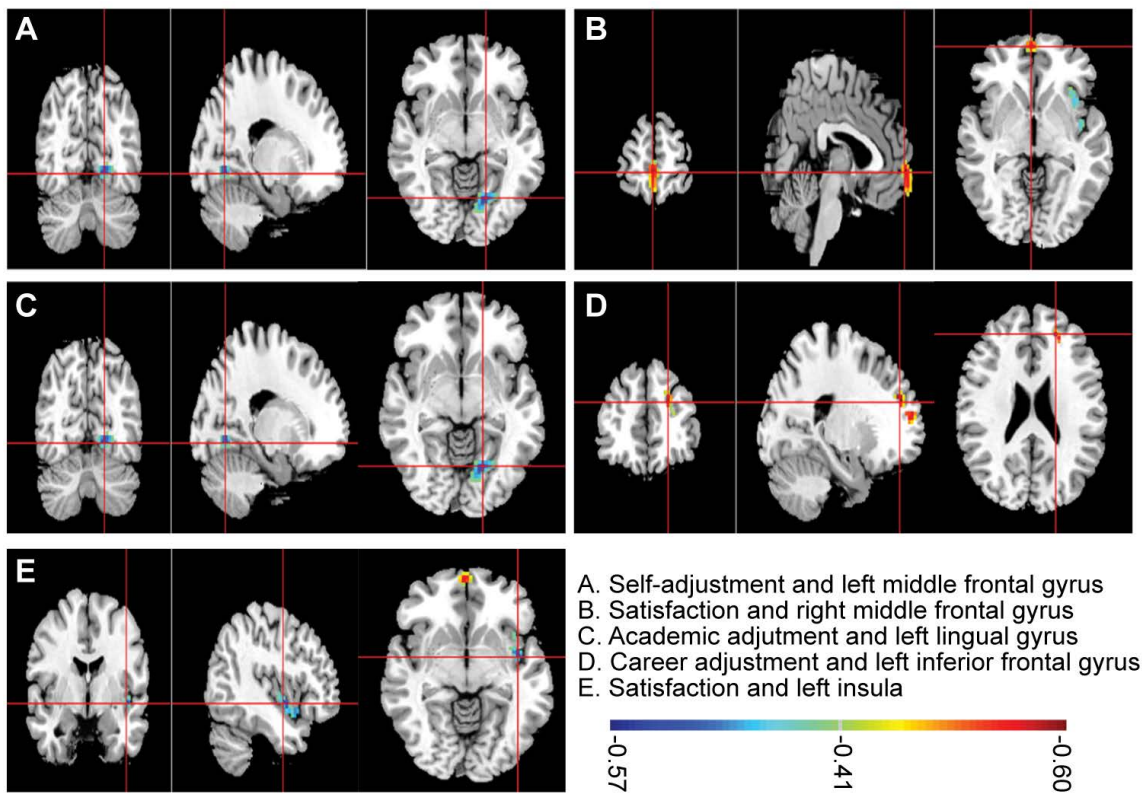

A. Self-adjustment and left middle frontal gyrus B. Satisfaction and right middle frontal gyrus

C. Academic adjutment and left lingual gyrus

D. Career adjustment and left inferior frontal gyrus

E. Satisfaction and left insula

\begin{tabular}{lll}
\hline 0 & $\dot{0}$ & $\dot{0}$
\end{tabular}

Figure 1. Brain regions show significant correlation of Reho value and CCSAS dimensions.
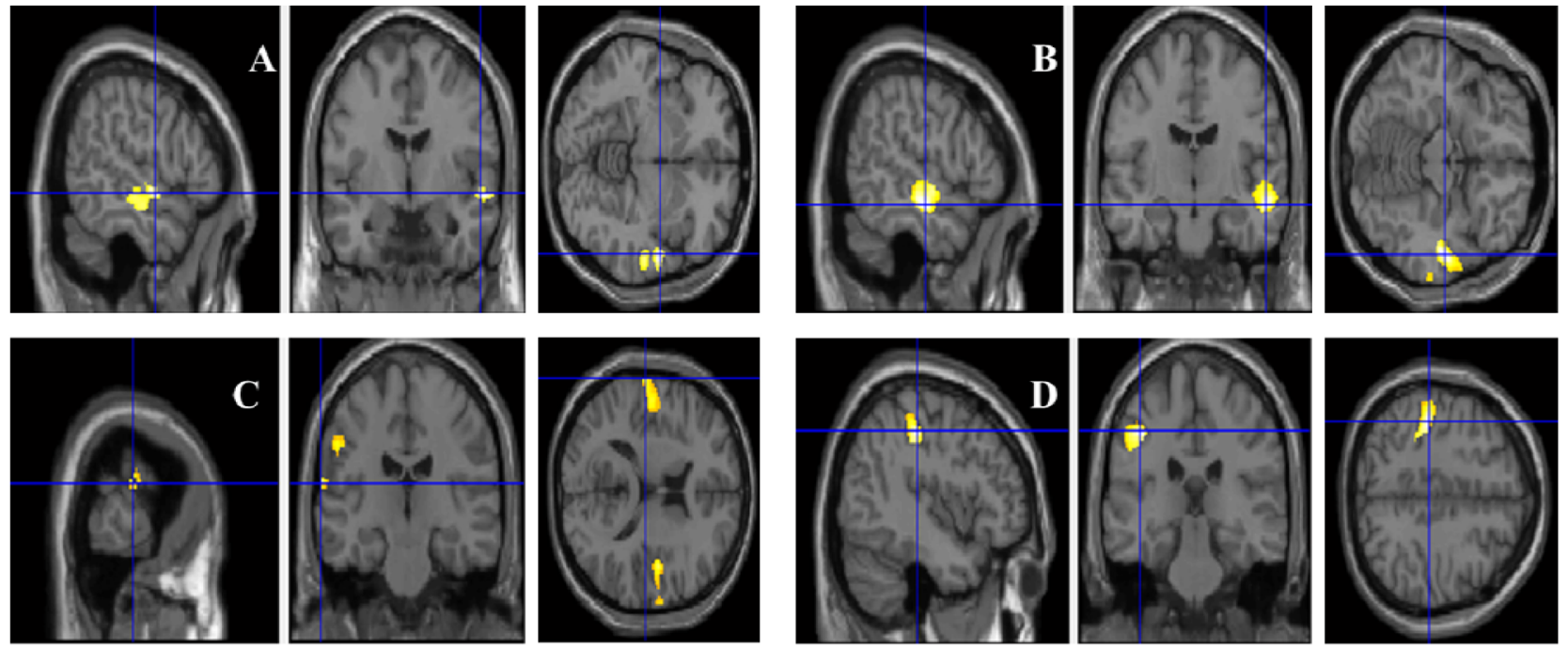

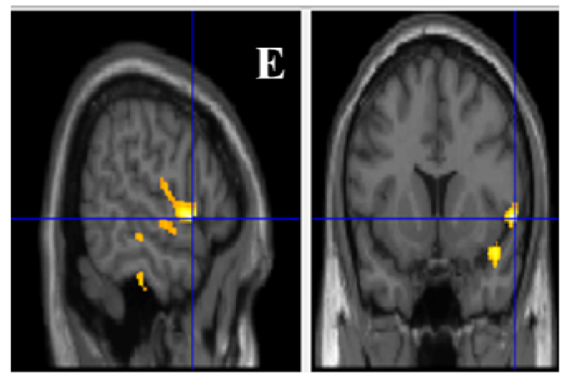

A. total score and the right superior temporal gyrus C. emotional adjustment and the left posterior central gyrus

E. satisfaction and the right superior temporal gyrus

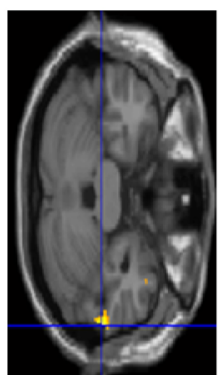

○

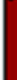

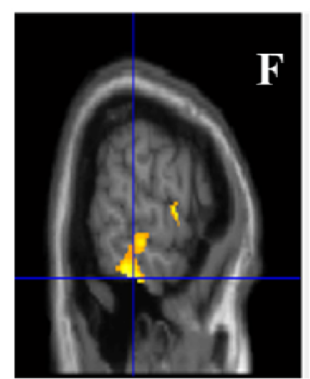

N
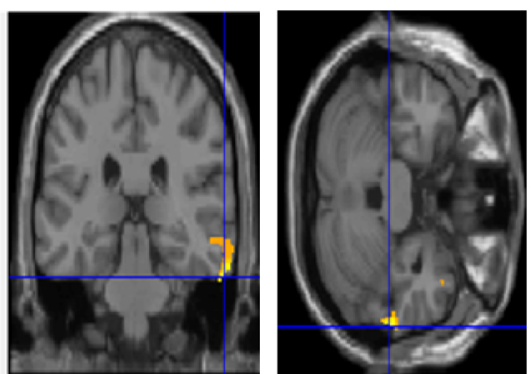

B. campus life adjustment and the right middle temporal gyrus

D. emotional adjustment and the left inferior

parietal lobe

F. satisfaction and the right middle temporal gyrus

Figure 2. Brain regions in which the grey matter density is significantly correlated with CCSAS dimensions. 
activity in the middle frontal gyrus [30]. The ReHo value of some regions in the left middle frontal gyrus decreases in patients with generalized anxiety disorder [31], and the self-rating score for depression is negatively correlated with the ReHo value of the right middle frontal gyrus [32]. These results indicate that the activation of the frontal gyrus decreases in patients with adaptation and psychiatric disorders, reflecting impaired prefrontal function, and further suggesting that activation of the frontal gyrus would help to improve self-adjustment and satisfaction. Career adjustment refers to the individuals' determination of their career goals according to their abilities, interests, and needs of the community. This adjustment includes active preparation for striving toward goals, including selection, planning, and decision-making processes. The inferior frontal gyrus is involved in cognitive control and risk-averse behavior, and can inhibit making high-risk choices [33] [34]. In the current study, the negative correlation between career adjustment and the inferior frontal gyrus reflects that inhibition by the inferior frontal gyrus enables individuals to make rational judgments and choices in the process of choosing careers, which includes setting reasonable goals and avoiding risks.

In the occipital lobe, academic adjustment was negatively correlated with the left lingual gyrus. Learning activities include integrative processing of language, memory, and attention. Academic adjustment refers to the mental and behavioral processes that individuals adopt according to requirements of the learning environment, and thereby attain balance within the academic environment. The occipital lobe is a part of the visual system, whereas the lingual gyrus is associated with visuospatial imagery and episodic memory extraction, and also has a role in processing some aspects of language and abstract concepts. In addition, the occipital lobe is involved in the regulation of inhibitory reaction [35]. Raichle et al. [36] found that normally, in the resting state, the occipital lobe shows negative activation (i.e., suppression), indicating that during learning, well-adapted individuals can distinguish between truth and falsehood, effectively prevent and inhibit certain negative or invalid types of stimulus, and rationally allocate mental resources, thus maximizing learning effects. Internet addicts and patients with adaptation disorder all exhibit excessive activation of the lingual gyrus and the occipital lobe, which induces overcompensation of inhibitory function [37] [38]. This further indicates that negative activation of the lingual gyrus enables effective learning activities.

Satisfaction is negatively associated with the left insula. The insula is located deep inside the cortex, and connects to many brain regions; it is thus critical in multimodal information integration. The insula regulates the balance among experience, emotion, and behavior [39], and is primarily involved in coding negative information such as hot and cold sensations, pain, hunger, nausea, and shame. The insula's activity is closely associated with the degree of an individual's desire [40] [41], and occurs in response to potential hazards and during an individual's reward selection [42]. That is, when individuals feel inadequate or 
unbalanced, the insula will be activated, whereas when individuals are satisfied, the metabolic activity of the insula will be reduced. Several studies have also shown that in patients with anxiety, depression, stress disorders, and addiction, the insula is abnormally hyperactive, which affects the processing and regulation of individuals' cognitive style and emotions [39] [43] [44] [45]. Thus, the insula can represent the achievement of physical satisfaction.

\subsection{Brain Structural Characteristics of Social Adjustment}

In the temporal lobe, the gray matter volume of the right superior temporal gyrus was positively correlated with the total social adjustment and satisfaction score, whereas the right middle temporal gyrus was positively correlated with measurements of campus life adjustment and satisfaction. The temporal lobe is a part of the prefrontal cortical network, which controls executive functions, and is primarily responsible for language comprehension, audiovisual information processing, proprioception, memory formation, and mood regulation [46]. The superior temporal gyrus contains the primary auditory cortex and auditory-related regions, and plays an important role in emotion and social cognition [47] [48]. The middle temporal gyrus is involved in the identification and perception of complex emotions in social situations, and with processing self-related information [49]. Social adjustment refers to the efforts of individuals to reach a state of harmony with the surrounding environment through social interaction. This includes changing themselves to adapt to the environment and changing the environment to better suit the individual. Campus life adjustment is necessary if students are to be happy with campus life and fulfill the tasks required of them as college students [10]. Studies have found that abnormal gray matter volume in the temporal lobe is associated with a variety of mental disorders. For example, gray matter volume is decreased as compared with controls in the superior temporal gyrus in patients with bipolar disorder [50] [51], in the superior and middle temporal gyrus in patients with schizophrenia [52] [53], in the middle temporal gyrus in patients with temporal lobe epilepsy [54] [55], and in the middle temporal gyrus in patients with alexithymia [56]. This indicates that disorders of the temporal lobe can result in a decline in cognitive function, including with respect to self-comprehension and memory. Therefore, the current study's positive correlation involving temporal lobe gray matter structure suggests that individuals with more gray matter volume in the temporal lobe quickly and accurately perceive links between themselves and the surrounding environment, and implement any necessary plans and tasks while in a pleasant emotional state, obtained through appropriate social interactions.

In the parietal lobe, measurements of emotional adjustment were positively correlated with the gray matter density of the left posterior central gyrus and the left inferior parietal lobule. The parietal cortex plays an important role in attention and sensory integration. As one of the main functional regions of the parietal lobe, the posterior central gyrus participates in attention and response in- 
hibition. If this brain region is dysfunctional, attention deficits and inappropriate inhibition will occur [57]. Compared with neurotypical individuals, patients with temporal lobe epilepsy and mild cognitive disorders exhibit varying degrees of gray matter density reduction in the posterior central gyrus [58] [59]. The inferior parietal lobule plays roles in focus maintenance, monitoring the environment for important stimuli, and self-identification [60], and is involved in individual implementation and cooperation, social cognition (i.e., understanding other's task-related behavior), and working memory [38]. Upon damage, attention is more easily distracted, and alertness is decreased. Related studies have found that the density of gray matter in the inferior parietal lobule decreases in patients with schizophrenia compared with controls [61] [62]. Emotional adjustment consists of an individual adjusting or maintaining their emotions to obtain a balanced and harmonious state with the environment in which the emotions occur. The positive correlation between emotional adjustment and the gray matter density in the posterior central gyrus and inferior parietal lobule may indicate that an individual can effectively maintain adaptability and coordination of emotions only by suppressing behaviors that do not meet the current needs, appropriately inhibiting responses as an important manifestation of executive function, and maintaining a dynamic equilibrium with the surrounding environment.

\section{Conclusions}

In this study, we assessed resting-state functional magnetic resonance imaging, structural magnetic resonance imaging, and behavioral measurements of social adjustment. The specific outcomes included the following:

1) Regarding brain function, some dimensions of social adjustment were associated with the insula, the frontal, and occipital lobes. Self-adjustment and satisfaction required the activation of the middle frontal gyrus, while career adjustment and academic adjustment required inhibition of the inferior frontal gyrus and lingual gyrus, respectively. A decrease in the metabolic activity of the insula was beneficial for achievement of satisfaction.

2) Regarding brain structure, the total social adjustment score and scores for some social adjustment dimensions were associated with gray matter structure in some regions of the temporal lobe and parietal lobe. For example, the superior temporal gyrus was correlated with the total score for social adjustment and for satisfaction, the middle temporal gyrus was correlated with campus life adaptation and with satisfaction, and the posterior central gyrus and inferior parietal lobule were correlated with emotional adaptation. To a certain extent, changes in the gray matter volume of these brain regions reflect the status of socially adaptive behaviors.

\section{Ethical Approval}

All procedures performed in studies involving human participants were in ac- 
cordance with the ethical standards of the institutional research committee of Chongqing University of Arts and Sciences and with the 1964 Helsinki declaration and its later amendments or comparable ethical standards.

\section{Informed Consent}

Informed consent was obtained from all individual participants included in the study.

\section{Conflict of Interest}

The authors declare that they have no conflict of interest.

\section{Acknowledgements}

This work was supported by the Key Project of Key Research Base for Humanities and Social Sciences of Chongqing (13SKB016).

\section{References}

[1] Zhang, C. (1992) Zhang's Psychology Dictionary. Shanghai Lexicographic Publishing House, Shanghai.

[2] Montgomery, E. and Foldspang, A. (2008) Discrimination, Mental Problems and Social Adaptation in Young Refugees. European Journal of Public Health, 18, 156-161. https://doi.org/10.1093/eurpub/ckm073

[3] Kolaitis, G., Tsiantis, J., Madianos, M. and Kotsopoulos, S. (2013) Psychosocial Adaptation of Immigrant Greek Children from the Former Soviet Union. European Child \& Adolescent Psychiatry, 12, 67-74. https://doi.org/10.1007/s00787-003-0310-x

[4] Bhattacharga, G. (2000) The School Adjustment of South Asian Immigrant Children in the U.S. Adolescence, 35, 77-85.

[5] Black, A.E. and Deci, E.C. (2000) The Effects of Instructors, Autonomy Support and Students. Autonomous Motivation on Learning Organic Chemistry: A Self-Determination Theory Perspective. Science Education, 84, 740-756. https://doi.org/10.1002/1098-237X(200011)84:6<740::AID-SCE4>3.0.CO;2-3

[6] Gong, C. and Li, C. (2012) Analysis and Countermeasures for Campus Adaptation of College Freshmen. Youth Exploration, 171, 42-47.

[7] Jiang, Q. and Xu, N. (2010) Effect of Coping Style and Social Adjustment on Mental Health of Undergraduates. Chinese Journal of Health Statistics, 27, 25-27.

[8] Li, C. and Zhou, W. (2009) A Research on the Relationship between Social Adjustment of College Students and Five-Factor Personality. Chinese Journal of Clinical Psychology, 17, 78-80.

[9] Si, X. and Li, H. (2008) Designing of Freshmen's Role Adjustment Questionnaire. Journal of Shenyang Normal University, 11, 64-67.

[10] Liu, Y. (2014) A Study on Campus Adaptation of Minority College Students. Master's Thesis, Beijing Technology and Business University, Beijing.

[11] Zou, X. (2013) A Study on Undergraduates' Adjustment to College in China. Master's Thesis, Xiamen University, Xiamen.

[12] Zang, Y., Jiang, T., Lu, Y., He, Y. and Tian, L. (2004) Regional Homogeneity Ap- 
proach to fMRI Data Analysis. NeuroImage, 22, 394-400.

https://doi.org/10.1016/j.neuroimage.2003.12.030

[13] Ashburner, J. and Friston, K.J. (2000) Voxel-Based Morphometry-The Methods. NeuroImage, 11, 805-851. https://doi.org/10.1006/nimg.2000.0582

[14] Goodadnd, C., Ashburner, J. and Frackowiak, R.S. (2001) Computational Neuroanatomy: New Perspectives for Neuroradiology. Revue Neurologique (Paris), 157, 797-806.

[15] Hahn, T., Dresler, T., Ehlis, A.C., Pyka, M., Dieler, A.C., Saathoff, C., Fallgatter, A.J., et al. (2012) Randomness of Resting-State Brain Oscillations Encodes Gray's Personality Trait. NeuroImage, 59, 1842-1845. https://doi.org/10.1016/j.neuroimage.2011.08.042

[16] Gao, Q., Wu, Q., Duan, X.J., Liao, W., Zhang, Z., Li, Y., Chen, H., et al. (2013) Extraversion and Neuroticism Relate to Topological Properties of Resting-State Brain Networks. Frontiers in Human Neuroscience, 7, 257.

[17] Aghajani, M., Veer, I.M., van Tol, M.J., Aleman, A., van Buchem, M.A., Veltman, D.J., van der Wee, N.J., et al. (2014) Neuroticism and Extraversion Are Associated with Amygdala Resting-State Functional Connectivity. Cognitive, Affective, \& Behavioral Neuroscience, 14, 836-848. https://doi.org/10.3758/s13415-013-0224-0

[18] Xu, J. and Potenza, M.N. (2012) White Matter Integrity and Five-Factor Personality Measures in Healthy Adults. NeuroImage, 59, 800-807.

https://doi.org/10.1016/j.neuroimage.2011.07.040

[19] Coutinho, J., Sampaio, A., Ferreira, M., Soares, J. and Goncalves, O. (2013) Brain Correlates of Pro-Social Personality Traits: A Voxel-Based Morphometry Study. Brain Imaging and Behavior, 7, 293-299. https://doi.org/10.1007/s11682-013-9227-2

[20] Liu, W.-Y., Weber, B., Reuter, M., Markett, S., Chu, W.-C. and Montag, C. (2013) The Big Five of Personality and Structural Imaging Revisited: A VBM-DARTEL Study. Neuroreport, 24, 375-380. https://doi.org/10.1097/WNR.0b013e328360dad7

[21] Fang, X., Wo, J. and Lin, X. (2005) The Development of "Chinese College Student Adaptation Scale". Studies of Psychology and Behavior, 3, 95-101.

[22] Wang, Z., Yan, C., Zhao, C., Qi, Z., Zhou, W., Lu, J. and Li, K. (2011) Spatial Patterns of Intrinsic Brain Activity in Mild Cognitive Impairment and Alzheimer's Disease: A Resting-State Functional MRI Study. Human Brain Mapping, 32, 1720-1740. https://doi.org/10.1002/hbm.21140

[23] Yan, C.G., Wang, X.D., Zuo, X.N. and Zang, Y.F. (2016) DPABI: Data Processing \& Analysis for (Resting-State) Brain Imaging. Neuroinformatics, 14, 339-351. https://doi.org/10.1007/s12021-016-9299-4

[24] Ashbumer, J. (2007) A Fast Diffeomorphic Image Registration Algorithm. NeuroImage, 55, 95-113. https://doi.org/10.1016/j.neuroimage.2007.07.007

[25] Abutalebi, J., Della Rosa, P.A., Green, D.W., Hernandez, M., Scifo, P., Keim, R. and Costa, A. (2012) Bilingualism Tunes the Anterior Cingulate Cortex for Conflict Monitoring. Cerebral Cortex, 22, 2076-2086. https://doi.org/10.1093/cercor/bhr287

[26] Frodl, T., Koutsouleris, N., Bottlender, R., Born, C., Jäger M., Mörgenthaler, M., Meisenzahl, E.M., et al. (2008) Reduced Gray Matter Brain Volumes Are Associated with Variants of the Serotonin Transporter Gene in Major Depression. Molecular Psychiatry, 13, 1093-1101. https://doi.org/10.1038/mp.2008.62

[27] Ashbumer, J. and Friston, K.J. (2005) Unified Segmentation. NeuroImage, 26, 839-851. https://doi.org/10.1016/j.neuroimage.2005.02.018

[28] Bryant, D.M., Hoeft, F., Lai, S., Lackey, J., Roeltgen, D., Ross, J. and Reiss, A.L. 
(2011) Neuroanatomical Phenotype of Klinefelter Syndrome in Childhood: A Voxel-Based Morphometry Study. The Journal of Neuroscience, 31, 6654-6660. https://doi.org/10.1523/JNEUROSCI.5899-10.2011

[29] Tregellas, J.R., Ellis, J., Shatti, S., Du, Y.P. and Rojas, D.C. (2009) Increased Hippocampal, Thalamic, and Prefrontal Hemodynamic Response to an Urban Noise Stimulus in Schizophrenia. American Journal of Psychiatry, 166, 354-360. https://doi.org/10.1176/appi.ajp.2008.08030411

[30] Wu, Q., Li, H., Xiao, H., Xiao-yang, W. and Bai-qian, C. (2015) An Investigation on the Regional Homogeneity in Resting-State fMRI in the Patients with Recruits' Adjustment Disorder. Functional and Molecular Medical Imaging (Electronic Edition), 4, 712-716.

[31] Zhang, H., Ran, S. and Li, H. (2015) Regional Homogeneity of Brain in Generalized Anxiety Disorder: A Resting State Functional MRI Study. Radiology Practice, 29, 1387-1391.

[32] Guo, W.B., Sun, X.L., Liu, L., Xu, Q., Wu, R.R., Liu, Z.N., Zhao, J.P., et al. (2011) Disrupted Regional Homogeneity in Treatment-Resistant Depression: A Resting-State fMRI Study. Progress in Neuro-Psychopharmacology \& Biological Psychiatry, 35, 1297-1302. https://doi.org/10.1016/j.pnpbp.2011.02.006

[33] Krain, A.L., Wilson, A.M., Arbuckle, R., Castellanos, F.X. and Milham, M.P. (2006) Distinct Neural Mechanisms of Risk and Ambiguity: A Meta-Analysis of Decision-Making. NeuroImage, 32, 477-484. https://doi.org/10.1016/j.neuroimage.2006.02.047

[34] Cox, C.L., Gotimer, K., Roy, A.K., Castellanon, F.X., Milham, M.P., Kelly, C., et al. (2010) Your Resting Brain CAREs about Your Risky Behavior. PLoS ONE, 5, e12296. https://doi.org/10.1371/journal.pone.0012296

[35] Haldane, M., Cunningham, G., Androutsos, C. and Frangou, S. (2008) Structural Brain Correlates of Response Inhibition in Bipolar Disorder. Journal of Psychopharmacology, 22, 138-143. https://doi.org/10.1177/0269881107082955

[36] Raichle, M.E., MacLeod, A.M., Snyder, A.Z., Powers, W.J., Gusnard, D.A. and Shulman, G.L. (2001) A Default Mode of Brain Function. Proceedings of the National Academy of Sciences USA, 98, 676-682. https://doi.org/10.1073/pnas.98.2.676

[37] Qin, L., Zhou, Y., Zhao, Z., Lu, Q., Ge, Z., Li, L., Gui, Y. and Xu, J. (2011) Preliminary Study of Brain Activity in Internet Addiction Adolescents: Revealed by Resting State Functional MRI. Journal of Clinical Radiology, 30, 7-10.

[38] Wu, D., Loke, I.C., Xu, F. and Lee, K. (2011) Neural Correlates of Evaluations of Lying and Truth-Telling in Different Social Contexts. Brain Research, 1389, 115-124. https://doi.org/10.1016/j.brainres.2011.02.084

[39] Nagai, M., Kishi, K. and Kato, S. (2007) Insular Cortex and Neuropsychiatric Disorders: A Review of Recent Literature. European Psychiatry, 22, 387-394. https://doi.org/10.1016/j.eurpsy.2007.02.006

[40] Franklin, T.R., Wang, Z., Wang, J., Sciortino, N., Harper, D., Li, Y., Childress, A.R., et al. (2007) Limbic Activation to Cigarette Smoking Cues Independent of Nicotine Withdrawal: A Perfusion fMRI Study. Neuropsychopharmacology, 32, 2301-2309. https://doi.org/10.1038/sj.npp.1301371

[41] Wang, Z., Faith, M., Patterson, F., Tang, K., Kerrin, K., Wileyto, E.P. and Lerman, C. (2007) Neural Substrates of Abstinence-Induced Cigarette Cravings in Chronic Smokers. The Journal of Neuroscience, 27, 14035-14040. https://doi.org/10.1523/JNEUROSCI.2966-07.2007

[42] Nakic, M., Smith. W., Busis, S., Vythilingam, M. and Blair, R.J.R. (2006) The Impact 
of Affect and Frequency on Lexical Decision: The Role of the Amygdala and Inferior Frontal Cortex. NeuroImage, 31, 1752-1761. https://doi.org/10.1016/j.neuroimage.2006.02.022

[43] Nasur, H.N. and Antoine, B. (2009) The Hidden Island of Addiction: The Insula. Trends in Neurosciences, 32, 56-67. https://doi.org/10.1016/j.tins.2008.09.009

[44] Ding, J. (2010) Magnetic Resonance Imaging Case Control Study on Brain Structural and Functional Abnormalities of First-Episode Medication-Naïve Adolescents with Major Depressive Disorder. Master D. Thesis, Central South University, Changsha.

[45] Zhang, X. (2014) The Magnetic Resonance Imaging Study on Brain Structure and Resting-State Function in Depressive Patients. PhD Thesis, Central South University, Changsha.

[46] Kalat, J.W. (2009) Biological Psychology. 10th Edition, Cengage Learning, Wadsworth.

[47] Allison, T., Puce, A. and McCarthy, G. (2000) Social Perception from Visual Cues: Institutional Role of the STS Region. Trends in Cognitive Science, 4, 267-278. https://doi.org/10.1016/S1364-6613(00)01501-1

[48] Gallagher, H.L. and Frith, C.D. (2003) Functional Imaging of Theory of Mind. Trends in Cognitive Science, 7, 77-83. https://doi.org/10.1016/S1364-6613(02)00025-6

[49] Viard, A., Piolino, P., Desgranges, B., Chételat, G., Lebreton, K., Landeau, B. and Eustache, F. (2007) Hippocampal Activation for Autobiographical Memories over the Entire Lifetime in Healthy Aged Subjects: An fMRI Study. Cerebral Cortex, 17, 2453-2467. https://doi.org/10.1093/cercor/bhl153

[50] Selvaraj, S., Amone, D., Job, D., Stanfield, A., Farrow, T.F., Nugent, A.C., McIntosh, A.M., et al. (2012) Grey Matter Differences in Bipolar Disorder: A Meta-Analysis of Voxel-Based Morphometry Studies. Bipolar Disorders, 14, 135-145. https://doi.org/10.1111/j.1399-5618.2012.01000.x

[51] Cui, L., Deng, W., Li-jun, J., Zhao-hua, H., Zhuang-fei, C., Ming-li, L., Tao, L., et al. (2010) Comparative Analysis of Brain Grey Matter Volume between Patients with Paranoid Schizophrenia and Patients with Bipolar Mania. Journal of Sichuan University (Medical Science Edition), 41, 5-9.

[52] Cobia, D.J., Smith, M.J., Wang, L. and Csernansky, J.G. (2012) Longitudinal Progression of Frontal and Temporal Lobe Changes in Schizophrenia. Schizophrenia Research, 139, 1-6. https://doi.org/10.1016/j.schres.2012.05.002

[53] Yüksel, C., McCarthy, J., Shinn, A., Pfaff, D.L., Baker, J.T, Heckers, S., Ongür, D., et al. (2012) Gray Matter Volume in Schizophrenia and Bipolar Disorder with Psychotic Features. Schizophrenia Research, 138, 177-182.

https://doi.org/10.1016/j.schres.2012.03.003

[54] Sandok, E.K., O’Brien, T.J., Jack, C.R. and So, E.L. (2000) Significance of Cerebellar Atrophy Intractable Temporal Lobe Epilepsy: A Quantitative MRI Study. Epilepsia, 41, 1315-1320. https://doi.org/10.1111/j.1528-1157.2000.tb04611.x

[55] Yu, A., Li, K., Li, L., Bao-ci, S. and Yu-ping, W. (2008) Whole-Brain Voxel-Based MRI Morphometric Study of Grey Matter in Medical Temporal Lobe Epilepsy. Chinese Journal of Medical Imaging Technology, 24, 1011-1014.

[56] Ihme, K., Dannlowski., U., Lichev, V., Stuhrmann, A., Grotegerd, D., Rosenberg, N., Suslow, T., et al. (2013) Alexithymia Is Related to Differences in Gray Matter Volume: A Voxel-Based Morphometry Study. Brain Research, 1491, 60-67. https://doi.org/10.1016/j.brainres.2012.10.044 
[57] Zuo, J., Xiong, J., Zhou, B. and Wang, B. (2015) Study of Functional Magnetic Resonance Imaging at Resting State for Patients in Sub-Health Status. Journal of Biomedical Engineering, 32, 635-639.

[58] Wu, X. (2014) The Voxel-Based Morphometry Research of Grey and White Matter in Patients with Temporal Lobe Epilepsy. Master D. Thesis, Xiamen University, Xiamen.

[59] Liu, H., Zhao, X., Wang, X., Le-le, Z. and Xi, Y. (2015) Grey Matter Volume in Mild Cognitive Impairment Patients: A Longitudinal Study Based on VBM. Journal of Tongji University (Medical Science), 36, 53-56.

[60] Lawrence, E.J., Shaw, P., Giampietro, V.P., Surguladze, S., Brammer, M.J. and David, A.S. (2006) The Role of "Shared Representations" in Social Perception and Empathy: An fMRI Study. NeuroImage, 29, 1173-1184.

https://doi.org/10.1016/j.neuroimage.2005.09.001

[61] Van Haren, N.E., Schnack, H.G., Cahn, W., van den Heuvel, M.P., Lepage, C., Collins, L., Kahn, R.S., et al. (2011) Changes in Cortical Thickness during the Course of Illness in Schizophrenia. Archives of General Psychiatry, 68, 871-880. https://doi.org/10.1001/archgenpsychiatry.2011.88

[62] Heuser, M., Thomann, P.A., Essig, M., Bachmann, S. and Schröder, J. (2011) Neurological Signs and Morphological Cerebral Changes in Schizophrenia: An Analysis of NSS Subscales in Patients with First Episode Psychosis. Psychiatry Research Neuroimaging, 192, 69-76. https://doi.org/10.1016/j.pscychresns.2010.11.009 